\title{
Performance Evaluation of a Color Sorter for Processsing Fresh Wild Blueberries
}

\author{
Travis J. Esau ${ }^{1 *}$, Aitazaz A. Farooque ${ }^{2}$, Farhat Abbas ${ }^{2}$ \\ ${ }^{1}$ Engineering Department Faculty of Agriculture, Dalhousie University, Truro, Canada \\ ${ }^{2}$ Faculty of Sustainable Design Engineering, University of Prince Edward Island, Charlottetown, Canada
}

\begin{abstract}
Rising production cost and declining farm gate price have decreased the profit margins for wild blueberry growers in Atlantic Canada. To date, the large majority (>95\%) of harvested wild blueberries are processed and sold as a frozen fruit product. Wild blueberry farmers in Nova Scotia are looking toward expanding the existing higher-value fresh fruit market to increase profit margins. Traditional equipment used for fresh wild blueberry processing is highly labour intensive and requires round the clock personnel to operate seasonally during a very short harvesting season. Advanced color sorting technologies have been developed for the fresh processing of several fruits and vegetables, but little research has been done to evaluate mechanized sorters for wild blueberries. The objective of this project was to compare the ability of an automated optical sorter to remove unripe and overripe wild blueberries and debris as compared to a traditional manually operated processing line. The overarching goal of this project is to effectively grow the supply of quality fresh fruit to increase the value of wild blueberries while minimizing processing labor and costs. A scientific comparative analysis of the two fresh fruit processing methods was conducted to aid wild blueberry farmers, processors and stakeholders in making informed decisions when pursuing options for processing wild blueberries for fresh market. Results from this study suggest that the automated equipment was able to significantly reduce pick over line labor requirements while achieving reasonable accuracy results. The color sorter removed an average of $52.43 \%$ of the foreign debris and unripe berries while mistargeting $2.3 \%$ good berries. Further evaluation is suggested using different combinations or additional auto sorters in parallel to determine if increased labor savings can be achieved. It would be valuable to understand any potential benefits from effectively pre-cooling berries prior to sorting in conjunction with monitoring the moisture content. Further research is also suggested to determine the effectiveness of the automatic sorters with berries that are mechanically harvested or raw supply (berries/debris) that were previously frozen.
\end{abstract}

